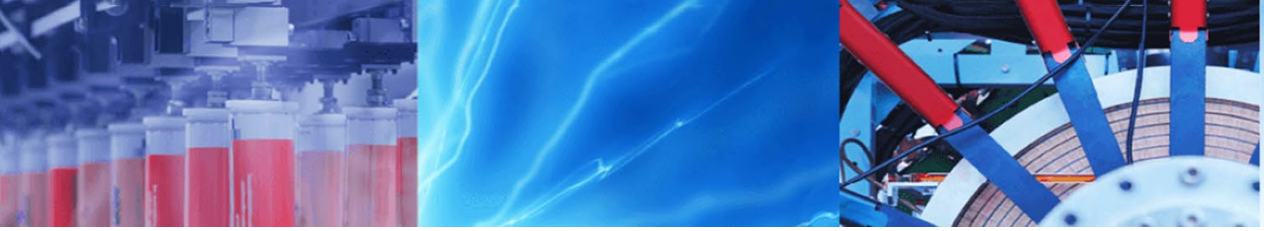

Research Article

\title{
Energy-efficient hybrid MAC protocol for railway monitoring sensor network
}

\author{
Manoj Tolani ${ }^{1}$ D $\cdot$ Sunny $^{2} \cdot$ Rajat Kumar Singh $^{2}$
}

Received: 16 April 2020 / Accepted: 8 July 2020 / Published online: 21 July 2020

(c) Springer Nature Switzerland AG 2020

\begin{abstract}
In the present work, a new protocol is proposed i.e. application-specific hybrid medium access control (ASHMAC) to efficiently handle data traffic generation in the railway track condition monitoring system. ASHMAC protocol is an improved version of the bit-map-assisted (BMA) medium access control (MAC) protocol by which it can efficiently handle continuous monitoring data traffic as well. Simulation and mathematical models have been developed and proposed for ASHMAC protocol. Its performance is compared with the other MAC protocols. The results reveal that the proposed protocol consumes $25-50 \%$ less energy w.r.t. time division multiple access (TDMA), energy-efficient TDMA, and BMA at low to medium data traffic conditions. For high data traffic conditions, the energy consumption of the proposed ASHMAC protocol is approximately equal to the other protocols. The transmission latency of ASHMAC is approximately $50 \%$ less than energy-efficient BMA. Both analytical and simulation results show the overall superiority of the proposed protocol.
\end{abstract}

Keywords Railway monitoring · Wireless sensor network · Railway sensor network · Medium access control ·

Transmission latency

\section{Introduction}

Recent advancement of the wireless sensor network (WSN) encourages to work in the field of railway track condition monitoring system (RTCMS). Specifically, this field can be called a railway sensor network (RSN). In recent years, increased demand for railway services has attracted the researchers to work in the field of RTCMS to maintain the railway services more secure, reliable, and safe [1-9]. A WSN can be efficiently used for railway monitoring application (RMA). The sensor network can be used to identify the major faults by continuously capturing the data from the railway track [1]. The data from the sensors may be generated continuously or periodically, or sometimes it can be event-driven. The sensor nodes which generate continuous monitoring data traffic, consume higher energy with respect to the event or periodic monitoring data traffic. Therefore, the data traffic generation in railway monitoring can be classified into two categories. First one is event-driven monitoring (e.g. pore pressure, temperature [2], water content [3]) and another one is continuous monitoring (e.g. acoustic emission [4, 5], accelerometer $[6,7]$ and strain gauge $[8,9])$. The energy demand of the sensor network is one of the major issues while designing and implementing WSN for RMA. The major sources of unnecessary energy consumption are idle listening, control frame overhead, overhearing, and collision [10]. Trans-receiver radio of sensor node operates in four different modes viz. transmit mode, receive mode, idle mode, and sleep mode [11]. Nodes should remain in sleep mode for the maximum duration so that their energy demand remains low. However, there is a trade-off between energy

\footnotetext{
$\triangle$ Manoj Tolani, rse2016004@iiita.ac.in; Sunny, sunnymeharwal@gmail.com; Rajat Kumar Singh, rajatsingh@iiita.ac.in | ${ }^{1}$ CC-3, 5228, Indian Institute of Information Technology-Allahabad, Prayagraj, India. ${ }^{2}$ CC-1, Indian Institute of Information Technology-Allahabad, Prayagraj, India.
} 
demand and the delay [12]. Various MAC protocols are proposed by the researchers to optimize the performance of WSN [10-17]. Broadly, the WSN MAC protocols are divided into two major categories i.e. contention-based MAC protocols and scheduled based MAC protocols. Contentionbased MAC protocols e.g. carrier sense multiple access (CSMA), consume more energy than scheduled based MAC protocols and are suitable for low and medium data traffic applications [18]. The major drawbacks of these protocols are collision, overhearing, and idle listening. Energy-efficient MAC protocols such as sensor MAC (S-MAC) and its variants work efficiently for WSN, but their latency is very high [10]. The improvements have been done by various researchers [12, 19-22]. However, they are suitable for very low data traffic applications. The good thing about the contention-based MAC protocols is that they have high scalability as compared to schedule based MAC protocols [18]. Schedule based MAC protocols are used for moderate to high traffic loads in a homogeneous network. It provides a collision-free environment and no overhearing. Various works have been reported for TDMA and its variants $[13,15,20,23]$. In most of the earlier reported works, the focus was to reduce energy demand and delay. In railway monitoring applications, few sensors generate continuous traffic when the train passes form the track (e.g. accelerometer). Hence, traffic is classified as medium to high traffic load as the data generated by the sensor at the rate of $25 \mathrm{kbps}$ [14]. BMA in its current form is not suitable for railway applications. It is only suitable for low to medium data traffic conditions. As per the application requirement, Shafiullah et al. proposed a new energy-efficient wireless MAC protocol E-BMA for railway monitoring application [14]. However, the major drawback of E-BMA MAC protocol is that the transmission latency of E-BMA is approximately two times higher w.r.t. TDMA, EA-TDMA, and BMA. Tolani et al. proposed adaptive-BMA (ABMA) MAC protocol to further reduce energy consumption. The energy consumption of $A B M A$ is less than E-BMA. However, the maximum transmission latency of $A B M A$ increases more than E-BMA. Therefore, both E-BMA and ABMA are not suitable for time-restrictive data traffic applications. In railway monitoring application, few sensors generate event-driven emergency data which requires the time-bound data transmission. To overcome the issue, in the present work, a new protocol is proposed for RMA. In the proposed protocol, the features of both TDMA and BMA have been merged that reduce energy consumption without compromising the maximum transmission latency. The proposed work concentrates on the major issue of handling the two types of traffic generation viz. continuous and event-driven/periodic monitoring. In RMA, when the train passes from the track, both continuous and event/periodic monitoring sensors are activated.
Otherwise, only event/periodic monitoring sensors are activated. Therefore, the traffic load changes dynamically from low-to-high and high-to-low. Our proposed ASHMAC protocol can handle such traffic load situations with low transmission latency as it includes the features of both TDMA and BMA. In addition, a comparison has been done among the proposed protocol and other MAC protocols that are dedicated to RMA for a real traffic environment. Both analytical and simulation models are developed for the proposed protocol to compare the performance with the existing MAC protocols.

The novelty of the proposed work can be defined as follows:

- A post-set-up-phase is introduced between the setup phase and the steady-state phase to characterize the sensor nodes into continuous and event-monitoring nodes. The post-set-up phase is a bit-map based arrangement used for characterizing the nodes.

- Unlike the BMA, E-BMA, TDMA, and EA-TDMA, in the proposed ASHMAC protocol, each session is divided into two sub-sessions.

- Sub-Session-1 follows TDMA for continuous monitoring nodes i.e. the nodes that utilize the slot in each session. Sub-session-2 follows BMA for event-monitoring nodes i.e. the nodes that not need slot in each session.

- The analytical model is developed for the performance analysis of the proposed ASHMAC protocol.

- The analytical model of TDMA, EA-TDMA, BMA, and E-BMA has been regenerated for the performance analysis of the continuous and event-monitoring data traffic generation.

- Unlike the previously reported works, in the proposed protocol, experimental analysis has been done using the prototype model (using Arduino and Raspberry-Pi Boards).

The rest of the paper is presented as follows. Section 2 describes the comparative study of scheduled based MAC protocol. Section 3 describes the problem statement. Section 4 discusses the proposed protocol. Section 5 discusses analytical and simulation modeling. Section 6 discusses the results and performance analysis. Finally, Sect. 7 concludes the major research findings of the proposed work.

\section{Comparative study of schedule based MAC protocols}

Various scheduled based MAC protocols have been discussed in this section for comparative analysis of our proposed protocol. G. Pei et al. presented a low power TDMA protocol for large WSN [13]. There are two common phases 
in cluster-based MAC protocols. The first phase is the setup phase or cluster formation phase and the second one is a steady-state phase as shown in Fig. 1. TDMA is suitable for high traffic conditions. For low data traffic conditions, TDMA suffers the serious drawback of inefficient energy consumption of non-source devices i.e. the devices that have no data to transmit.

To overcome the problems of TDMA, J. Li et al. presented bit-map-assisted (BMA) energy-efficient MAC protocol, which performs better for low and medium traffic conditions [16]. BMA is a schedule based MAC protocol, which reduces the unnecessary energy consumption of non-source node and cluster head $(\mathrm{CH})$ node (i.e. energy wastage due to idle listening). The operational diagram of $B M A$ is shown in Fig. 1. The set-up phase of BMA is similar to the TDMA protocol whereas the steady-state phase is quite different from TDMA [13]. The steady-state phase starts with a contention access period, which is further divided into mini control slots. Each sensor node (SN) gets its mini control slot and transmits a 1-bit control message to the $\mathrm{CH}$ node during its allotted time slot. BMA consists of a larger contention period as compared to TDMA, which causes unnecessary overhead at high traffic loads where the data transmission is almost certain. Therefore, for high data traffic applications, BMA is not a suitable protocol.

To avoid the unnecessary wastage of energy due to idle listening, Shafiullah et al. [15] developed an energyefficient EA-TDMA protocol. EA-TDMA operational diagram is shown in Fig. 1. In this protocol, every node wakes-up in its allotted time slot and checks its buffer. If the buffer is empty, the node turns 'OFF' its radio immediately and goes in sleep mode, which saves fair amount of energy. However, the $\mathrm{CH}$ keeps its radio turn 'ON' for the full duration. Hence, the major energy wastage occurs due to the $\mathrm{CH}$ node. To overcome the contention overhead problem due to high data traffic conditions in BMA, Shafiullah et al. proposed a new energy-efficient wireless MAC protocol named E-BMA [14] for RMA. E-BMA consumes less energy than BMA even at high data traffic load conditions. However, the maximum transmission latency of E-BMA is approximately two times higher w.r.t. BMA, EA-TDMA, and TDMA. Therefore, E-BMA is not suitable for time-constraint data traffic applications. The operational diagram of E-BMA is shown in Fig. 1. Ayona et al. proposed a time-adaptive bit-map-assisted (TA-BMA) MAC protocol to make E-BMA more energy efficient. However, TA-BMA compromises with the transmission latency [25]. Alvi et al. proposed a scalable energy-efficient TDMA based MAC protocol to handle diverse data traffic, but the performance is not compared with E-BMA protocol [23]. Debasis et al. proposed the bit-map-assisted energy-efficient medium access control (BEE-MAC) protocol to reduce energy consumption [26]. In the BEE-MAC protocol, the transmission schedule is broadcasted in each round, which reduces the energy consumption. However, BEE-MAC also compromises with the maximum transmission latency [26]. Saurabh et al. proposed a hybrid bitmap-assisted MAC protocol for the vehicular ad-hoc network (VANET) to reduce the packet overhead by using fixed-size bitmap [30]. Tolani et al. proposed the ABMA MAC protocol in

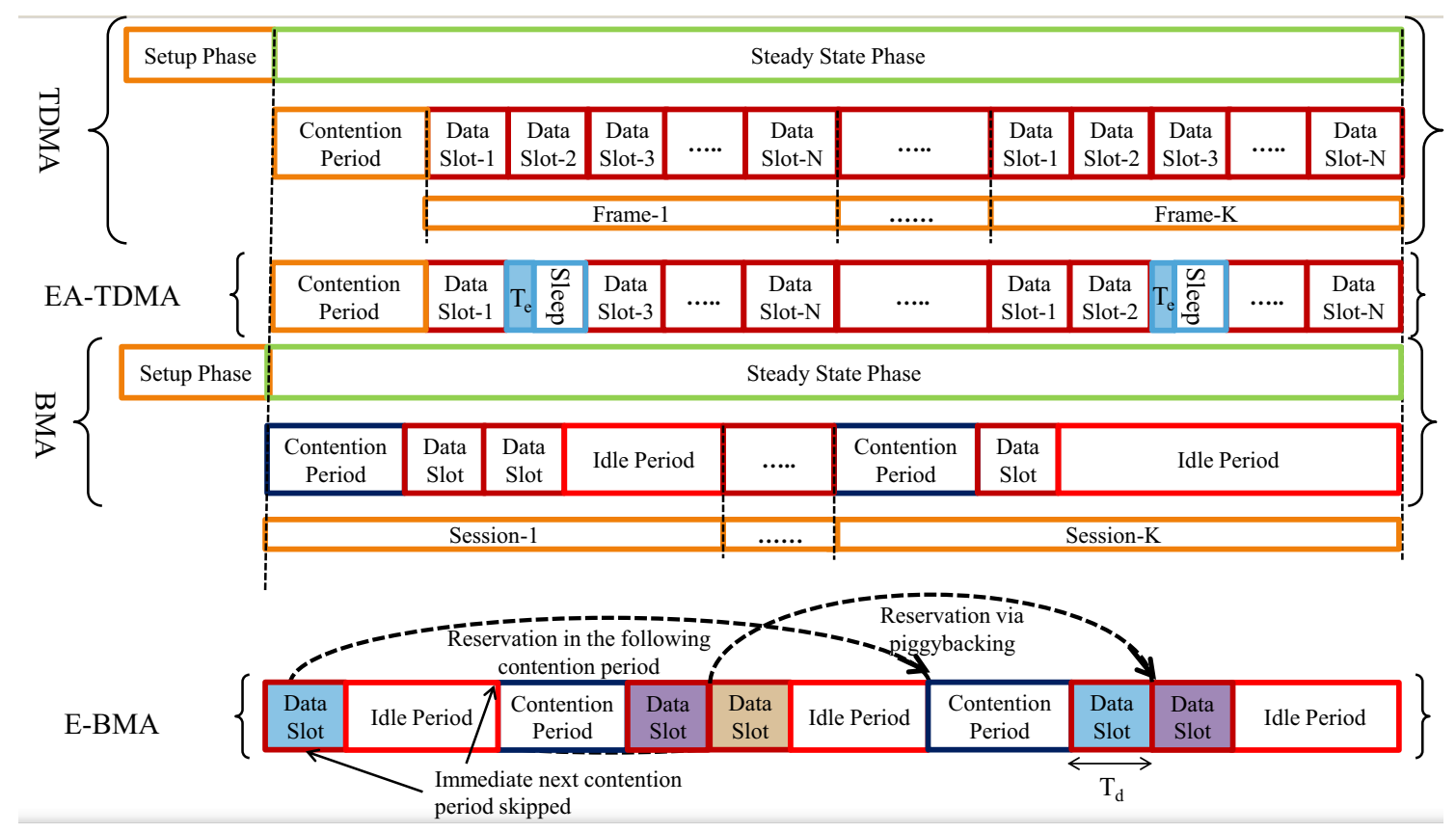

Fig. 1 Operational Diagram of TDMA [13], EA-TDMA [15], BMA [16] and E-BMA [14] 
which two bits control field is used for the reservation of data slots and piggybacking [27]. ABMA reduces energy consumption, but it further increases the maximum transmission latency more than E-BMA. Tolani et al. proposed the duty cycle enabled energy-efficient bit-map-assisted (DCE-EBMA) MAC protocol in which adaptive duty cycle method is used for E-BMA MAC protocol to further reduce energy consumption [28]. However, duty cycle method increases the maximum transmission latency multiple times. To overcome the issues, we have proposed a new energy-efficient hybrid MAC protocol (ASHMAC) that has been discussed in Sect. 4.

\section{Problem statement}

As per the railway application requirement, TDMA and EATDMA are suitable for high traffic conditions or continuous monitoring data traffic applications. On the other hand, BMA performs better for event-driven data traffic applications $[16,18,23,27]$. However, in WSN based railway monitoring environment, the sensor nodes generate both types of traffic [31]. Both types of sensors (continuous and event-driven) are controlled by the same cluster head. In these types of railway traffic situations, none of the TDMA, EA-TDMA, and BMA performs optimally. BMA wastes bit mapping period unnecessarily for the continuous monitoring nodes. The performance of TDMA and EA-TDMA degrades due to event-driven nodes, where non-source event-driven nodes waste time slot unnecessarily in idle listening. The operational diagram of TDMA, EA-TDMA, $B M A$, and E-BMA are shown in Fig. 1.

Although, E-BMA and ABMA reduces energy consumption but compromises with maximum transmission latency and are not suitable for time-constraint data traffic transmission. In the present work, we have proposed a hybrid protocol that handles such real traffic situations more efficiently than the existing MAC protocols for RMA. In the proposed protocol, which is an extension of $B M A$, different types of traffic generating nodes are handled separately. Each session is divided into two sub-sessions i.e. Sub-Session-1 and Sub-Session-2 as shown in Fig. 2. The former is reserved for continuous monitoring nodes and the latter is reserved for event-driven nodes. The detailed discussion of the proposed protocol has been done in section 4. Our contribution to the present work can be summarized as follows:

- A hybrid protocol is proposed i.e. ASHMAC, to efficiently handle low, medium, and high traffic load conditions for RMA.

- ASHMAC handles continuous-monitoring and eventmonitoring nodes separately in an energy-efficient manner, which improves its performance w.r.t. other protocols.

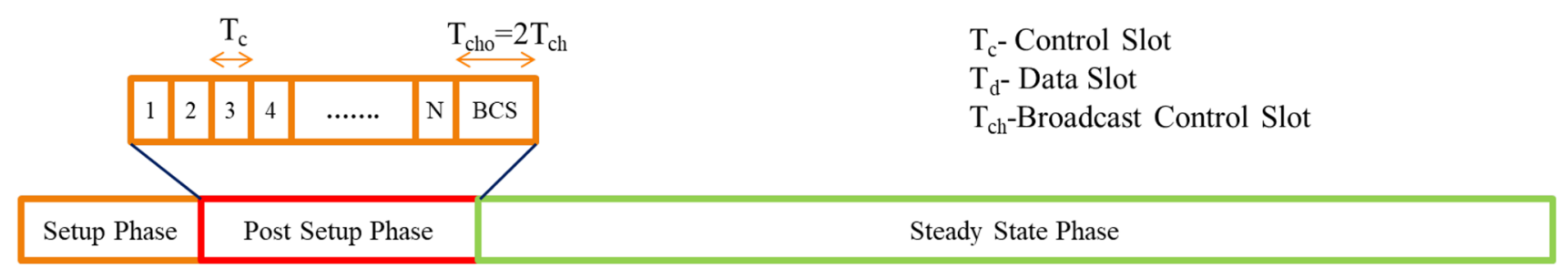

$\mathrm{T}_{\mathrm{c}}-$ Control Slot

$\mathrm{T}_{\mathrm{d}^{-}}$- Data Slot

$\mathrm{T}_{\mathrm{ch}}$-Broadcast Control Slot

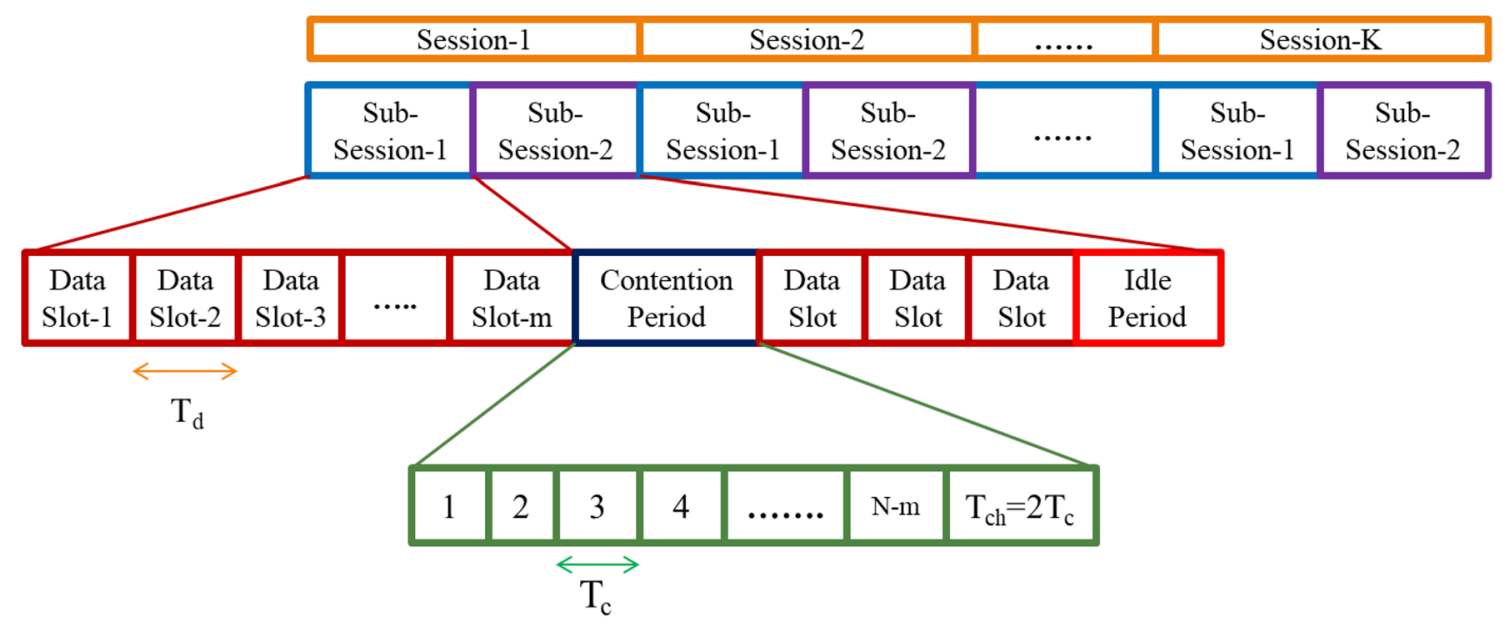

Fig. 2 Operational diagram of ASHMAC 
- The proposed protocol reduces energy-consumption and transmission latency by optimum utilization of the channel.

- The proposed protocol can handle dynamic changes more efficiently i.e. low-to-high and high-to-low traffic load with low transmission latency.

- The comparison of the proposed protocol with other MAC protocols has been done in terms of energy consumption and maximum transmission latency.

\section{Application specific hybrid MAC protocol}

The operational diagram of the proposed ASHMAC protocol is shown in Fig. 2. The operation can be divided into multiple rounds. TDMA and BMA features are used for continuous monitoring nodes and event-driven nodes respectively. For this purpose, $\mathrm{CH}$ categorizes the nodes into event-driven and continuous monitoring nodes. A new phase is introduced between the set-up and steady-state phase called the post-setup phase. Both cluster formation and $\mathrm{CH}$ selection take place in the set-up phase. The operation of the set-up phase is similar to the LEACH (low energy adaptive clustering hierarchy) protocol [17]. In the post-set-up phase, all the nodes keep their radios ' $\mathrm{ON}^{\prime}$ and wait for their scheduled slot. These are bit-map-assisted slots. Each node gets its specific slot and transmits a 1-bit control message in its scheduled slot if it is a continuous monitoring node. Otherwise, the scheduled slot remains empty. After getting the information from all $N$ devices, the $\mathrm{CH}$ has complete knowledge of continuous monitoring and event-driven nodes. $\mathrm{CH}$ broadcasts the information of sub-frame allotment in each session and allows the slots to the continuous monitoring nodes. The operation is divided into the multiple rounds and each round consists of $k$ sessions. Each session is further divided into two subsessions. The first sub-session (Sub-Session-1) is allotted to the continuous monitoring nodes and second sub-session (Sub-Session-2) is allotted to the event-driven nodes. Continuous monitoring nodes get their scheduled slot allotment in the post-set-up phase. Similar to the TDMA, there is no repeated contention phase in a single round for continuous monitoring nodes. Each node wakes up in its scheduled time slot. The node transmits the data and stays in sleep mode for the remaining duration of the session. Therefore, Sub-Session-1 has only data slots for continuous monitoring nodes without the contention phase. Sub-Session-2 is allotted for event-driven nodes. In each session, the Sub-Session-2 is started with the contention phase. Similar to the BMA, if the node has data to transmit then all the event-driven nodes keep their radios 'ON' during contention phase and transmit 1-bit control message in its scheduled slot. Otherwise, its scheduled slot remains empty. An event-driven node, which has data to transmit, is called a source node. After the contention phase, $\mathrm{CH}$ has complete information about the nodes that have data to transmit. The $\mathrm{CH}$ broadcasts a transmission schedule for the source nodes. After receiving $\mathrm{CH}$ message, each node wakes up in its scheduled slot and transmits the data. For the rest of the session, they turn 'OFF' their radio and remain in sleep mode. After the data transmission of all event-driven source nodes, system proceeds in the idle period and all the devices turn 'OFF' their radio during this period. Similar to the BMA, a data transmission period can be varied in different sessions. However, combined sum of data transmission period and the idle period is fixed and constant. Therefore, the size of Sub-Session- 2 is fixed which implies that the size of the complete session is also fixed. Finally, after completion of the session, a new session is started with Sub-Session- 1 and the whole process is repeated. Analytical and Simulation models are developed and analyzed for TDMA [13], EA-TDMA [15], BMA [16], E-BMA [14] and our proposed protocol ASHMAC. Energy consumption and latency are considered as the performance parameter.

\section{Analytical and simulation modeling}

To prove the superiority of ASHMAC, we have compared its performance with the existing wireless MAC protocols for RMA [14]. The analytical model of TDMA, EA-TDMA, $B M A$, and $E-B M A$ are regenerated for the present scenarios [13-16]. It is assumed that a cluster network has already been formed. Energy consumption and latency are analyzed for all other phases excluding the set-up phase. numbers of nodes are considered in WSN where one of them is $\mathrm{CH}$ and all remaining are sensor nodes (i.e. non$\mathrm{CH}$ ). If non- $\mathrm{CH}$ nodes are denoted by $N$, then $\aleph=N+1$. Out of $N$ non- $\mathrm{CH}$ nodes, $m$ nodes are continuous monitoring nodes and rest $(\mathrm{N}-\mathrm{m})$ nodes are event-driven nodes. Continuous monitoring nodes generate real-time traffic whereas event-driven nodes generate traffic only at the specific occurrence of an event. Therefore, it is uncertain that SNs have data to transmit in every session or frame. In a WSN scenario, it is assumed that there are $k$ sessions and in a specific session, there are $n$ source nodes, which generate event-driven traffic out of the total $(N-m)$ eventdriven nodes. The remaining nodes are non-source nodes. Thus, at any event, if a node has data to transmit then it can be considered as Bernoulli trial [16]. If $p$ is the probability of a node having data to transmit, then the number of event-driven source nodes is given by

$n=(N-m) p$ 
The analytical model of TDMA, EA-TDMA, BMA, and E-BMA are already derived in the previously reported works [13-16]. However, for the comparison of the proposed protocol with previously reported works, the scenarios are considered for event-driven and continuous monitoring applications. Therefore, the analytical model of TDMA, EATDMA, BMA, and E-BMA are regenerated for our proposed analysis. Energy consumption and latency analysis for all protocols i.e. TDMA, EA-TDMA, BMA, E-BMA, and ASHMAC are discussed in the next subsections. All the parameters used in the analysis are given in Table 1.

\subsection{TDMA protocol}

\subsubsection{Energy consumption of TDMA protocol}

TDMA cycle starts with contention period during which all the nodes keep their radio ' $\mathrm{ON}$ ' and $\mathrm{CH}$ assigns a time slot for data transmission to all the non- $\mathrm{CH}$ nodes. $\mathrm{CH}$ broadcasts a control message and all the nodes receive it. Therefore, energy consumption by $\mathrm{CH}$ node is $P_{t} T_{c}$ and total energy consumption by non- $\mathrm{CH}$ nodes is $N P_{r} T_{c}$. Energy consumption in contention period is given by

Table 1 Symbols used in analysis of WSN scenario

\begin{tabular}{ll}
\hline Symbol & Quantity \\
\hline$N$ & Total no. of nodes in cluster \\
$N$ & Total no. of nodes in cluster excluding $\mathrm{CH}$ \\
$m$ & Total no. of continuous monitoring nodes \\
$N-m$ & Total no. of event monitoring nodes \\
$n$ & Total no. of event monitoring source nodes \\
$P_{t}$ & Transmit power consumption \\
$P_{r}$ & Receiving power consumption \\
$P_{i}$ & Idle power consumption \\
$P_{e}$ & Power consumption in checking buffer [15] \\
$T_{d}$ & Data slot time \\
$T_{c}$ & Control slot time \\
$T_{c h}$ & Cluster-head broadcast slot time \\
$T_{e}$ & The time required to switch on its device, \\
& check buffer and turn 'OFF' its radio [15] \\
$T_{c h o}$ & The broadcast time slot in post-set-up phase \\
$p$ & Traffic generation probability of a node \\
$k$ & Number of sessions/frames per round \\
\hline
\end{tabular}

$E_{\text {TDMA-CONT-Total }}=P_{t} T_{c}+N P_{r} T_{c}$

All the notations pertaining to TDMA are given in Table 2 . After contention period, the data transmission period starts which is further divided into frames. Each frame has $\mathrm{N}$ number of slots for $\mathrm{N}$ non- $\mathrm{CH}$ nodes. Each node transmits data in its allotted time slot in each frame, and during the rest of the period, its radio remains 'OFF'. Therefore, energy consumed by the $\mathrm{CH}$ node to receive the data packet is $P_{r} T_{d}$ and energy consumption to transmit the data packet by each node is $P_{t} T_{d}$. The continuous monitoring $m$ nodes generate traffic in each frame, whereas $n$ out of $(N-m)$ nodes generates event-driven traffic. So, the expected numbers of event-driven source nodes are $(N-m) p$ and event-driven non-source nodes are $(N-m)(1-p)$. Source nodes transmit the data packet and non-source nodes remain in idle state during their allotted slots. Therefore, the total expected energy consumption for transmission of packet by the non- $\mathrm{CH}$ nodes in each frame is given by, $\left(m P_{t} T_{d}+(N-m) p P_{t} T_{d}+(N-m)(1-p) P_{i} T_{d}\right)$ and total expected energy consumption for receiving the packets by $\mathrm{CH}$ node in each frame is given by, $\left(m P_{r} T_{d}+(N-m) p P_{r} T_{d}+(N-m)(1-p) P_{i} T_{d}\right)$. Total energy consumption in the transmission mode is given by

$$
\begin{aligned}
E_{\text {TDMA-TRANS-Total }}= & m P_{t} T_{d}+(N-m) p P_{t} T_{d} \\
& +(N-m)(1-p) P_{i} T_{d} \\
& +m P_{r} T_{d}+(N-m) p P_{r} T_{d} \\
& +(N-m)(1-p) P_{i} T_{d}
\end{aligned}
$$

In each round, there are $k$ frames. Therefore, average energy consumption per round in TDMA protocol is given by

$$
\begin{aligned}
E_{T D M A}= & P_{t} T_{c}+N P_{r} T_{c}+k\left(m P_{t} T_{d}\right. \\
& +(N-m) p P_{t} T_{d}+(N-m)(1-p) P_{i} T_{d} \\
& \left.+m P_{r} T_{d}+(N-m) p P_{r} T_{d}+(N-m)(1-p) P_{i} T_{d}\right)
\end{aligned}
$$

\subsubsection{Transmission latency of TDMA protocol}

Maximum transmission latency can be derived from the frame cycle as shown in Fig. 1, which is given by
Table 2 Symbols used in analytical modeling of TDMA

\begin{tabular}{ll}
\hline Symbol & Quantity \\
\hline$E_{\text {TDMA-CONT-Total }}$ & Total energy consumption in contention period for TDMA \\
$E_{\text {TDMA-TRANS-Total }}$ & Total energy consumption in transmission period for TDMA \\
$E_{T D M A}$ & Total energy consumption of TDMA \\
$L_{\text {TDMA }}$ & Maximum transmission latency of TDMA \\
\hline
\end{tabular}


$L_{T D M A}=\frac{T_{c}+N k T_{d}}{k}$

\subsection{EA-TDMA protocol}

\subsubsection{Energy consumption of EA-TDMA protocol}

Similar to the TDMA protocol, energy consumption of EATDMA protocol in contention period is given by,

$E_{E A-T D M A-C O N T-\text { Total }}=P_{t} T_{c}+N P_{r} T_{c}$

All the notations pertaining to EA-TDMA are given in Table 3.

Data transmission period starts after the contention period. In the data transmission period, each node wakes up in their scheduled time slot and checks their buffer. If the nodes have no data to transmit, they turn 'OFF' their radio immediately. Energy consumption of the nodes which have no data transmit is given by $P_{e} T_{e}$ (where $T_{e}<T_{d}$ ). Energy consumption of all other nodes is similar to the TDMA. Therefore, energy consumption of EA-TDMA is given by

$$
\begin{aligned}
E_{\text {EA-TDMA-TRANS-Total }}= & m P_{t} T_{d} \\
& +(N-m) p P_{t} T_{d}+(N-m)(1-p) P_{e} T_{e} \\
& +m P_{r} T_{d}+(N-m) p P_{r} T_{d} \\
& +(N-m)(1-p) P_{i} T_{d}
\end{aligned}
$$

In each round, there are $k$ frames. Therefore, average energy consumption per round in EA-TDMA protocol is given by

$$
\begin{aligned}
E_{E A-T D M A}= & P_{t} T_{c}+N P_{r} T_{c}+k\left(m P_{t} T_{d}+(N-m) p P_{t} T_{d}\right. \\
& +(N-m)(1-p) P_{e} T_{e}+m P_{r} T_{d}+(N-m) p P_{r} T_{d} \\
& \left.+(N-m)(1-p) P_{i} T_{d}\right)
\end{aligned}
$$

\subsubsection{Transmission latency of EA-TDMA protocol}

Maximum transmission latency of EA-TDMA is same as TDMA as shown in Fig. 1, which is given by

$L_{E A-T D M A}=\frac{T_{c}+N k T_{d}}{k}$

\subsection{BMA protocol}

\subsubsection{Energy consumption of BMA protocol}

In BMA, each cycle is divided into contention phase and data transmission phase. In contention phase, all the nodes turn 'ON' their radios. Contention phase of BMA is divided into $N$ control slots. Each source node transmit control packet in their scheduled slot and all other nodes remain in idle mode. The $m$ continuous monitoring nodes and $(N-m) p$ event monitoring nodes are source nodes. All the remaining nodes are non-source nodes. After the contention slots, $\mathrm{CH}$ broadcasts the information of the reserved data slots. Energy consumption of BMA during contention phase is given by

$$
\begin{aligned}
E_{\text {BMA-CONT-CH }}= & m P_{r} T_{c}+(N-m) p P_{r} T_{c} \\
& +(N-m)(1-p) P_{i} T_{c}+P_{t} T_{c h} \\
E_{\text {BMA-CONT-NCH }}= & N P_{r} T_{c h}+m\left(P_{t} T_{c}+(N-1) P_{i} T_{c}\right) \\
& +(N-m) p\left(P_{t} T_{c}+(N-1) P_{i} T_{c}\right) \\
& +(N-m)(1-p)\left(P_{i} T_{c}+(N-1) P_{i} T_{c}\right) \\
E_{\text {BMA-CONT-Total }}= & P_{t} T_{c h}+m P_{r} T_{c}+(N-m) p P_{r} T_{c} \\
& +(N-m)(1-p) P_{i} T_{c}+N P_{r} T_{c h} \\
& +m\left(P_{t} T_{c}+(N-1) P_{i} T_{c}\right) \\
& +(N-m) p\left(P_{t} T_{c}+(N-1) P_{i} T_{c}\right) \\
& +(N-m)(1-p)\left(P_{i} T_{c}+(N-1) P_{i} T_{c}\right)
\end{aligned}
$$

All the notations pertaining to BMA are given in Table 4 .

After the contention period, data transmission period starts. In data transmission period, each source node sends the data packet in their scheduled slot. All the non-source nodes keep their radio 'OFF' during this session.

$$
\begin{aligned}
E_{\text {BMA-TRANS-Total }}= & m P_{r} T_{d} \\
& +(N-m) p P_{r} T_{d}+m P_{t} T_{d}+(N-m) p P_{t} T_{d}
\end{aligned}
$$

Each round comprised of $k$ sessions, average energy consumption per round in BMA protocol is given by
Table 3 Symbols used in analytical modeling of EA-TDMA

\begin{tabular}{ll}
\hline Symbol & Quantity \\
\hline$E_{E A-T D M A-C O N T-T o t a l}$ & Total energy consumption in contention period for EA-TDMA \\
$E_{E A-T D M A-T R A N S-T o t a l}$ & Total energy consumption in transmission period for EA-TDMA \\
$E_{E A-T D M A}$ & Total energy consumption of EA-TDMA \\
$L_{E A-T D M A}$ & Maximum transmission latency of EA-TDMA \\
\hline
\end{tabular}


Table 4 Symbols used in analytical modeling of BMA

\begin{tabular}{ll}
\hline Symbol & Quantity \\
\hline$E_{B M A-C O N T-C H}$ & Energy consumption of $\mathrm{CH}$ in contention period for BMA \\
$E_{B M A-C O N T-N C H}$ & Energy consumption of Non-CH nodes in contention period for BMA \\
$E_{B M A-C O N T-T o t a l}$ & Total energy consumption in contention period for BMA \\
$E_{B M A-T R A N S-T o t a l}$ & Total energy consumption in transmission period for BMA \\
$E_{B M A}$ & Total energy consumption of BMA \\
$L_{B M A}$ & Maximum transmission latency of BMA \\
\hline
\end{tabular}

$$
\begin{aligned}
E_{B M A}= & \left(P_{t} T_{c h}+N P_{r} T_{c h}+m P_{r} T_{c}+(N-m) p P_{r} T_{c}\right. \\
& +(N-m)(1-p) P_{i} T_{c}+m\left(P_{t} T_{c}+(N-1) P_{i} T_{c}\right) \\
& +(N-m) p\left(P_{t} T_{c}+(N-1) P_{i} T_{c}\right) \\
& +(N-m)(1-p)\left(P_{i} T_{c}+(N-1) P_{i} T_{c}\right)+m P_{r} T_{d} \\
& \left.+(N-m) p P_{r} T_{d}+m P_{t} T_{d}+(N-m) p P_{t} T_{d}\right) k
\end{aligned}
$$

\subsubsection{Transmission latency of BMA protocol}

Maximum transmission latency can be derived from the frame cycle as shown in Fig. 1, which is given by

$L_{B M A}=N T_{c}+T_{c h}+N T_{d}$

\subsection{E-BMA protocol}

\subsubsection{Energy consumption of E-BMA protocol}

E-BMA cycle starts with the contention phase. Source nodes, which have not reserved data slot in their preceding data packet, they send a control message in their respective contention slot. The non-source nodes and piggybacked nodes keep their radios 'OFF' during the entire contention period. The probability of a data packet not being piggybacked is $p(1-p)$. Continuous monitoring nodes ( $m$ nodes) generate traffic in each session. Therefore, they are piggybacked in their preceding data slots by keeping their radios 'OFF' in their respective contention slots. Moreover, $\mathrm{CH}$ remains in idle mode during these contention slots. After the contention slots, $\mathrm{CH}$ broadcasts the information of reserved data slots to the nodes who had demanded the reservation. Energy consumption in a contention phase of E-BMA is given by

$$
\begin{aligned}
E_{E-B M A-C O N T-C H}= & m P_{i} T_{c}+(N-m) p(1-p) P_{r} T_{c} \\
& +(N-m)(1-p(1-p)) P_{i} T_{c}+P_{t} T_{c h} \\
E_{E-B M A-C O N T-N C H}= & N P_{r} T_{c h}+(N-m) p(1-p)\left(P_{t} T_{c}\right. \\
& \left.+(N-1) P_{i} T_{c}\right) \\
E_{E-B M A-C O N T-T o t a l}= & m P_{i} T_{c}+(N-m) p(1-p) P_{r} T_{c} \\
& +(N-m)(1-p(1-p)) P_{i} T_{c} \\
& +N P_{r} T_{c h}+(N-m) p(1-p)\left(P_{t} T_{c}\right. \\
& \left.+(N-1) P_{i} T_{c}\right)+P_{t} T_{c h}
\end{aligned}
$$

All notations pertaining to E-BMA are given in Table 5.

After the contention period, data transmission period starts. Each source node sends the data packet in their allotted data slot. All the non-source nodes keep their radio 'OFF' in session duration. As reported in the [14] that piggybacking requires only 1 -bit extra space in the data packet, which is negligible w.r.t. the packet size. The expected energy consumption in transmission is given by

$$
\begin{aligned}
E_{E-B M A-T R A N S-T o t a l}= & m P_{r} T_{d}+(N-m) p P_{r} T_{d} \\
& +m P_{t} T_{d}+(N-m) p P_{t} T_{d}
\end{aligned}
$$

Each round comprised of $k$ sessions, average energy consumption per round in E-BMA protocol is given by
Table 5 Symbols used in analytical modeling of E-BMA

\begin{tabular}{ll}
\hline Symbol & Quantity \\
\hline$E_{E-B M A-C O N T-C H}$ & Energy consumption of $\mathrm{CH}$ in contention period for E-BMA \\
$E_{E-B M A-C O N T-N C H}$ & Energy consumption of non-CH nodes in contention period for E-BMA \\
$E_{E-B M A-C O N T-T o t a l}$ & Total energy consumption in contention period for E-BMA \\
$E_{E-B M A-T R A N S-T o t a l}$ & Total energy consumption in transmission period for E-BMA \\
$E_{E-B M A}$ & Total energy consumption of E-BMA \\
$L_{E-B M A}$ & Maximum transmission latency of E-BMA \\
\hline
\end{tabular}




$$
\begin{aligned}
E_{E-B M A}= & \left(m P_{i} T_{c}+(N-m) p(1-p) P_{r} T_{c}\right. \\
& +(N-m)(1-p(1-p)) P_{i} T_{c}+N P_{r} T_{c h} \\
& +(N-m) p(1-p)\left(P_{t} T_{c}+(N-1) P_{i} T_{c}\right) \\
& +P_{t} T_{c h}+m P_{r} T_{d}+(N-m) p P_{r} T_{d} \\
& \left.+m P_{t} T_{d}+(N-m) p P_{t} T_{d}\right) k
\end{aligned}
$$

\subsubsection{Transmission latency of E-BMA protocol [14]}

Maximum transmission latency can be derived from the frame cycle as shown in Fig. 1. Each data packet has to wait for one additional frame duration before being transmitted. Therefore, the average maximum transmission latency is given by

$L_{E-B M A}=T_{c h}+2\left(T_{c}+T_{d}\right) N$

\subsection{ASHMAC protocol}

\subsubsection{Energy consumption of ASHMAC protocol}

A new phase is introduced in ASHMAC between steady state and set-up phase called post-set-up phase. In all the previous protocols, we had excluded the energy calculations of set-up phase. The same has been done here as well. After the set-up phase, post-set-up phase starts with mini control. Similar to the set-up phase, the post-set-up phase is also repeated after each round. All the devices turn 'ON' their radios during the post-set-up phase. Each device transmits control message in their scheduled time slot and remains in idle mode for $(N-1)$ slots. Control message consists of a flag bit. Continuous monitoring nodes set it to ' 1 ' and eventdriven nodes set it to ' 0 '. After completion of $N$ control slots,
$\mathrm{CH}$ has information of all the continuous monitoring and event-driven nodes. Therefore, $\mathrm{CH}$ broadcasts a message to all the devices for the separate sub-session division of the continuous monitoring and event-driven nodes. $\mathrm{CH}$ also provides the slot-allotment for Sub-Session- 1 to continuous monitoring $m$ nodes (see Fig. 2.). Energy consumption in post-set-up phase is given by

$E_{\text {ASHMAC-Post-Setup-CH }}=N P_{r} T_{c}+P_{t} T_{\text {cho }}$

$E_{\text {ASHMAC-Post-Setup-NCH }}=N\left(P_{t} T_{c}\right.$

$$
\left.+(N-1) P_{i} T_{c}+P_{r} T_{\text {cho }}\right)
$$

$E_{\text {ASHMAC-Post-Setup-Total }}=N P_{r} T_{c}+P_{t} T_{\text {cho }}+N\left(P_{t} T_{c}\right.$

$$
\left.+(N-1) P_{i} T_{c}+P_{r} T_{c h o}\right)
$$

All the notations pertaining to ASHMAC are given in Table 6.

After the post-set-up phase, steady-state phase starts which is further sub-divided into several sessions. Here, each session has two sub-sessions. In Sub-Session-1, each continuous monitoring node transmits its data to the $\mathrm{CH}$ node in its scheduled time slot. All the event-driven nodes keep their radio 'OFF' during the Sub-Session-1. Energy consumption in Sub-Session- 1 is given by

$$
\begin{aligned}
& E_{\text {ASHMAC-SS-1-CH }}=m P_{r} T_{d} \\
& E_{\text {ASHMAC-SS-1-NCH }}=m P_{t} T_{d} \\
& E_{\text {ASHMAC-SS-1-Total }}=m P_{t} T_{d}+m P_{r} T_{d}
\end{aligned}
$$

Table 6 Symbols used in analytical modeling of ASHMAC

\begin{tabular}{ll}
\hline Symbol & Quantity \\
\hline$E_{\text {ASHMAC-Post-Setup-CH }}$ & Energy consumption of CH in post setup-phase for ASHMAC \\
$E_{A S H M A C-P o s t-S e t u p-N C H}$ & Energy consumption of non-CH node in post-setup-phase for ASHMAC \\
$E_{\text {ASHMAC-Post-Setup-Total }}$ & Total energy consumption of nodes in post-setup-phase for ASHMAC \\
$E_{\text {ASHMAC-SS-1-CH }}$ & Energy consumption of CH in Sub-Session-1 for ASHMAC \\
$E_{\text {ASHMAC-SS-1-NCH }}$ & Energy consumption of non-CH node in Sub-Session-1 for ASHMAC \\
$E_{\text {ASHMAC-SS-1-Total }}$ & Total energy consumption of nodes in Sub-Session-1 for ASHMAC \\
$E_{\text {ASHMAC-SS-2-CONT-CH }}$ & Energy consumption of CH in contention phase for ASHMAC in Sub-Session-2 \\
$E_{\text {ASHMAC-SS-2-CONT-NCH }}$ & Energy consumption of non-CH nodes in contention phase for ASHMAC in Sub-Session-2 \\
$E_{\text {ASHMAC-SS-2-CONT-Total }}$ & Total energy consumption of nodes in contention phase for ASHMAC in Sub-Session-2 \\
$E_{A S H M A C-S S-2-T R A N S-C H}$ & Energy consumption of CH in transmission phase for ASHMAC in Sub-Session-2 \\
$E_{\text {ASHMAC-SS-2-TRANS-NCH }}$ & Energy consumption of non-CH in transmission phase for ASHMAC in Sub-Session-2 \\
$E_{A S H M A C-S S-2-T R A N S-T o t a l}$ & Total energy consumption in transmission phase for ASHMAC in Sub-Session-2 \\
$E_{A S H M A C}$ & Total energy consumption of ASHMAC \\
$L_{A S H M A C}$ & Maximum transmission latency of ASHMAC \\
\hline
\end{tabular}


Sub-Session-2 starts with contention phase similar to the BMA, which is repeated in each Sub-Session-2. All the continuous monitoring nodes keep their radios 'OFF' during Sub-Session-2. There are $(N-m)$ event-driven nodes. Therefore, contention phase consists of $(N-m)$ control slots. For the contention phase of Sub-Session-2, all eventdriven nodes turn 'ON' their radios. Each event-driven node transmits a control packet in their scheduled slot if they have data to transmit else they remain in idle mode. For the rest $(N-m-1)$ slots, source nodes remain in idle mode. Energy consumption in contention phase of SubSession-2 is given by

$$
\begin{aligned}
E_{A S H M A C-S S-2-C O N T-C H}= & (N-m) p P_{r} T_{c}+P_{t} T_{c h} \\
& +(N-m)(1-p) P_{i} T_{c} \\
E_{A S H M A C-S S-2-C O N T-N C H}= & (N-m) p\left(P_{t} T_{c}+(N-m-1) P_{i} T_{c}\right) \\
& +(N-m)(1-p)\left((N-m) P_{i} T_{c}\right) \\
& +(N-m) P_{r} T_{c h}
\end{aligned}
$$

$E_{\text {ASHMAC-SS-2-CONT-Total }}=(N-m) p P_{r} T_{c}+P_{t} T_{c h}$

$$
+(N-m) p\left(P_{t} T_{c}+(N-m-1) P_{i} T_{c}\right)
$$$$
+(N-m)(1-p)\left((N-m) P_{i} T_{c}\right)
$$$$
+(N-m) P_{r} T_{c h}+(N-m)(1-p) P_{i} T_{c}
$$

The data transmission period starts after the contention period, and each source node sends the data packet in its allotted data slot. All the non-source nodes keep their radios 'OFF' during a Sub-Session-2 similar to the BMA. Energy consumption in transmission phase of Sub-Session-2 is given by

$$
\begin{aligned}
& E_{\text {ASHMAC-SS-2-TRANS-CH }}=(N-m) p P_{r} T_{d} \\
& E_{\text {ASHMAC-SS-2-TRANS-NCH }}=(N-m) p P_{t} T_{d} \\
& E_{\text {ASHMAC-SS-2-TRANS-Total }}=(N-m) p P_{t} T_{d}+(N-m) p P_{r} T_{d}
\end{aligned}
$$

Each round comprised of $k$ sessions, average energy consumption per round in ASHMAC protocol is given by

$$
\begin{aligned}
E_{\text {ASHMAC }}= & N P_{r} T_{c}+P_{t} T_{c h o}+N\left(P_{t} T_{c}+(N-1) P_{i} T_{c}\right. \\
& \left.+P_{r} T_{c h o}\right)+k\left((N-m) p P_{r} T_{c}+P_{t} T_{c h}\right. \\
& +(N-m) p\left(P_{t} T_{c}+(N-m-1) P_{i} T_{c}\right) \\
& +(N-m)(1-p)\left((N-m) P_{i} T_{c}\right) \\
& +(N-m) P_{r} T_{c h}+(N-m)(1-p) P_{i} T_{c} \\
& \left.+(N-m) p P_{t} T_{d}+(N-m) p P_{r} T_{d}\right)
\end{aligned}
$$

\subsubsection{Transmission latency of ASHMAC protocol}

Maximum transmission latency can be derived from the frame cycle as shown in Fig. 2, which is given by

$L_{\text {ASHMAC }}=T_{c h}+(N-m) T_{c}+N T_{d}+\frac{N T_{c}+T_{c h o}}{k}$

\section{Results and performance analysis}

\subsection{Analytical and simulation analysis}

The performance of proposed protocol ASHMAC is compared with the other MAC protocols in terms of energy consumption and transmission latency. The ZigBee-enabled 2.4 GHz CC2420 RF transceiver [24, 29] is used for the analytical and simulation analysis. The comparison chart for different radios is given in Table 7. For $250 \mathrm{kbps}$ data rate the $C C 2420$ radio consumes less power for transmitting and receiving the packet. In addition, CC2420 radio is used as a standard radio in various simulation platforms e.g. Castalia (OMNet++), NetSim, NS2. The only drawback of CC2420 is that the idle power consumption of CC2420 is more than MC13202. For comparison of our proposed protocol with other protocols on same simulation platform, we have used the same energy node model as reported in [14]. The power consumption in transmitting, receiving and idle mode is $50 \mathrm{~mW}, 54 \mathrm{~mW}$ and $54 \mathrm{~mW}$, respectively as given in Table 7 [29]. These power ratings are comparable to CC2420 RF transceiver specification. The data rate is $25 \mathrm{kbps}$ and control packet size is 5 bytes as reported in [14]. For simplicity $T_{e}=T_{d} / 5, P_{e}=P_{i}$. Broadcast-controlslot in case of ASHMAC is larger $\left(T_{\text {cho }}=2 T_{c h}\right)$. Comparative
Table 7 Characteristics of typical radios used by the sensor nodes [29]

\begin{tabular}{llclll}
\hline & RFM TR 1000 & RFM TR 3000 & MC 13202 & CC 1000 & CC 2420 \\
\hline Data rate (kbps) & 115.2 & 115.2 & 250 & 76.8 & 250 \\
Transmit current $(\mathrm{mA})$ & 12 & 7.5 & 35 & 16.5 & 17.4 \\
Receive current $(\mathrm{mA})$ & 3.8 & 3.8 & 42 & 9.6 & 18.8 \\
Idle current $(\mathrm{mA})$ & 3.8 & 3.8 & 0.8 & 9.6 & 18.8 \\
Standby current $(\mu \mathrm{A})$ & 0.7 & 0.7 & 102 & 96 & 426 \\
\hline
\end{tabular}


analysis has done on MATLAB platform. For comparative analysis, five cases are considered for energy efficiency and two cases are considered for maximum transmission latency.

\subsubsection{Energy efficiency}

Case 1: In this case, analytical and simulation analysis has been done for all five protocols as mentioned above. In this scenario, the total number of nodes is $\aleph=15$ and non$\mathrm{CH}$ nodes are $\mathrm{N}=14$ (where four nodes are continuous monitoring nodes i.e. $m=4$ and rest are event monitoring nodes). Each round consists of 20 frames i.e. $k=20$, and data packet size is 200 bytes. In this case, the transmission probability of the event monitoring nodes is varying from 0.0 to 1.0. Energy consumption variation versus probability is plotted in Fig. 3. At $p=0$, no event monitoring node generates traffic i.e. $n=0$. In this case, the transmission traffic is only due to the continuous monitoring nodes $(m=4)$. The graph shown in Fig. 3 reveals that for $p<0.8$, our proposed protocol consumes less energy than EATDMA, TDMA, and BMA MAC protocols.

Case 2: In this case, it is assumed that $p=0.2, k=20$ frames/round and data packet size is 200 bytes. The total number of non- $\mathrm{CH}$ nodes are varied from $\mathrm{N}=7$ to 35 (where $m$ varied from 2 to 10 and rest event monitoring nodes varied from 5 to 25). Energy consumption versus total number of nodes is plotted in Fig. 4, which reveals that ASHMAC performs better than BMA, TDMA and EATDMA for different cluster sizes.

Case 3 : In this situation, it is assumed that the total number of non- $\mathrm{CH}$ nodes are $\mathrm{N}=14$, the probability of event generated traffic $p=0.2$ and data packet size is 200 bytes.

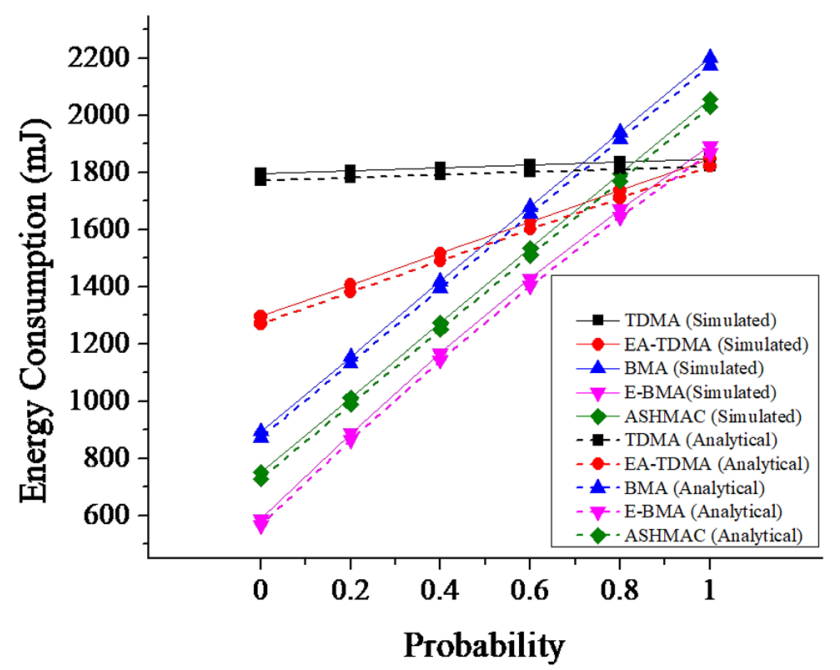

Fig. 3 Analytical and Simulation results of data generation probability versus energy consumption for $\mathrm{N}=14, \mathrm{k}=20$

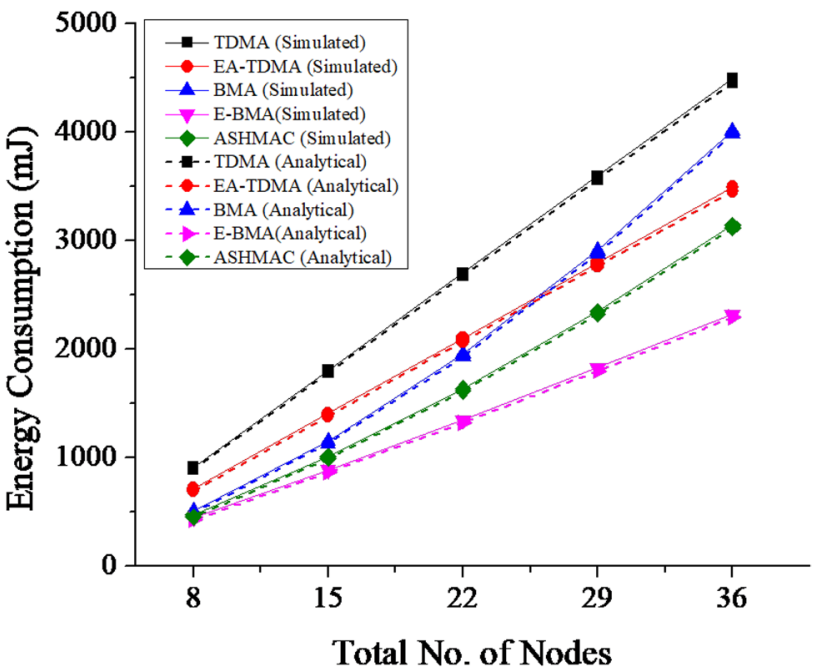

Fig. 4 Analytical and Simulation results of number of nodes versus energy consumption for $p=0.2, \mathrm{k}=20$

Number of frames per round $(k)$ is varied from 10 to 100 . Both analytical and simulation results show that ASHMAC consumes less energy than BMA, TDMA and EA-TDMA as shown in Fig. 5.

Case 4: In this experiment, it is presumed that the total number of non- $\mathrm{CH}$ nodes are $N=14$, the probability of event generated traffic $p=0.2$ and number of frames per round $(k)$ is 20 . Data packet size is varied from 50 to 250 bytes. Both analytical and simulation results show that ASHMAC consumes less energy than BMA, TDMA and EATDMA as shown in Fig. 6.

Case 5: In this case, continuous monitoring nodes vary whereas event-monitoring nodes remain fixed. This experiment is performed to analyze the performance of

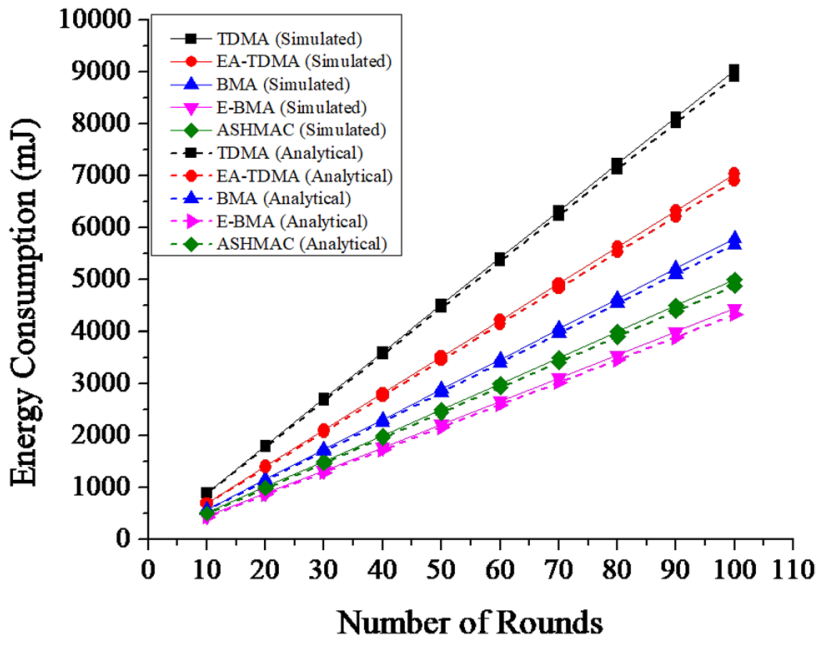

Fig. 5 Analytical and Simulation results of number of rounds versus energy consumption for $\mathrm{N}=14, p=0.2$ 


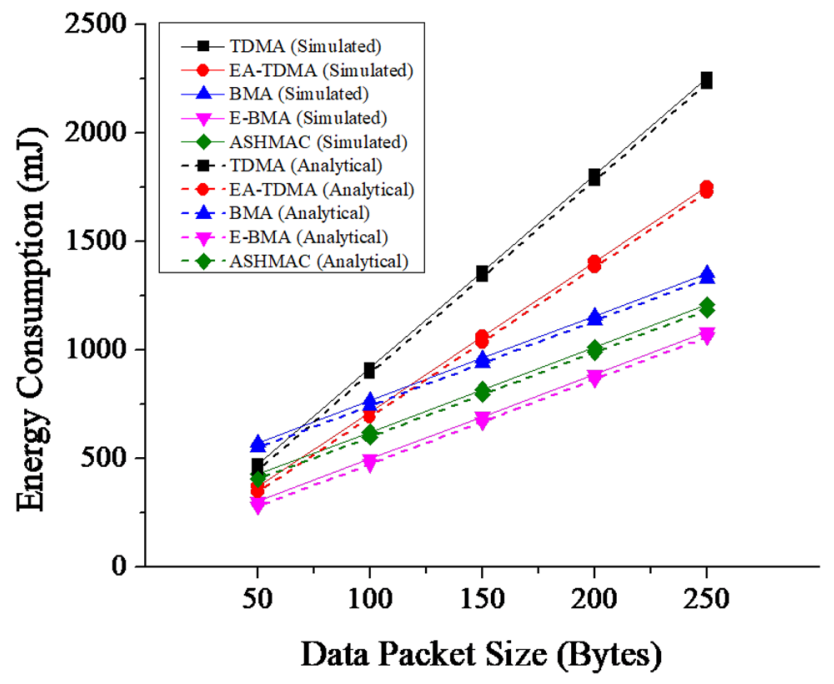

Fig. 6 Analytical and Simulation results of data packet size versus energy consumption for $\mathrm{N}=14, \mathrm{k}=20, p=0.2$

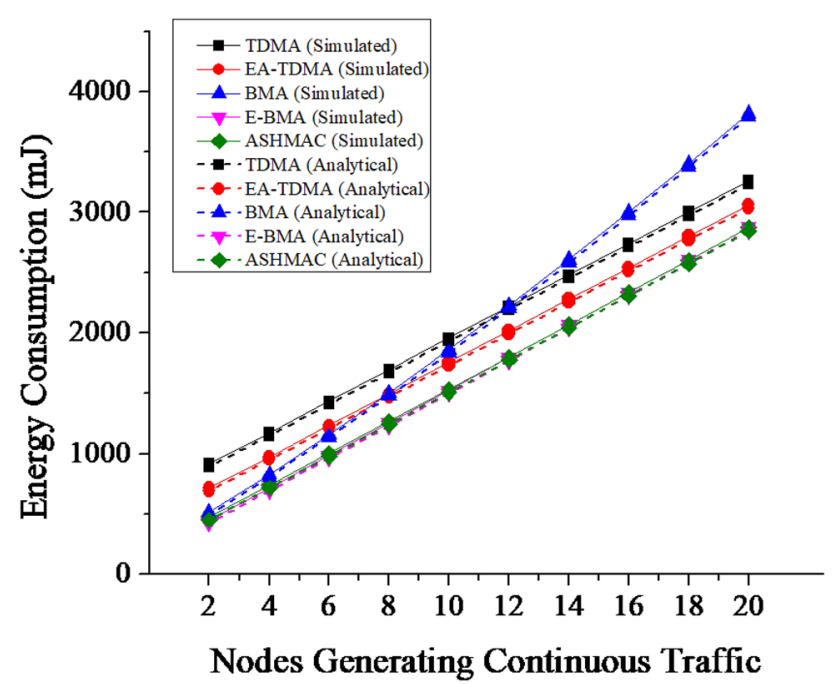

Fig. 7 Analytical and simulation results of no. of continuous monitoring nodes versus energy consumption

heavy traffic load due to continuous monitoring nodes. Therefore, the total number of non- $\mathrm{CH}$ nodes is varied from $N=7$ to 15 (where $m$ varied from 2 to 10 ). For this experiment, $p=0.2$, data packet size is 200 bytes, $k=20$ frames/round. The performance is analyzed for different cluster sizes and different data packet sizes. Analytical and simulation results show that ASHMAC consumes less energy than TDMA, EA-TDMA, BMA and equivalent to E-BMA as shown in Fig. 7.

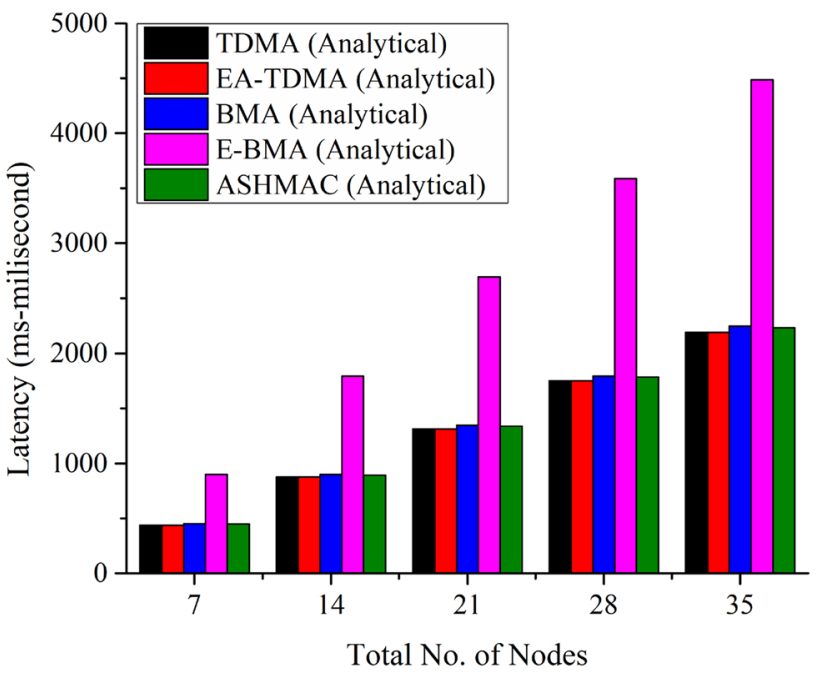

Fig. 8 Analytical results of transmission latency versus total number of nodes

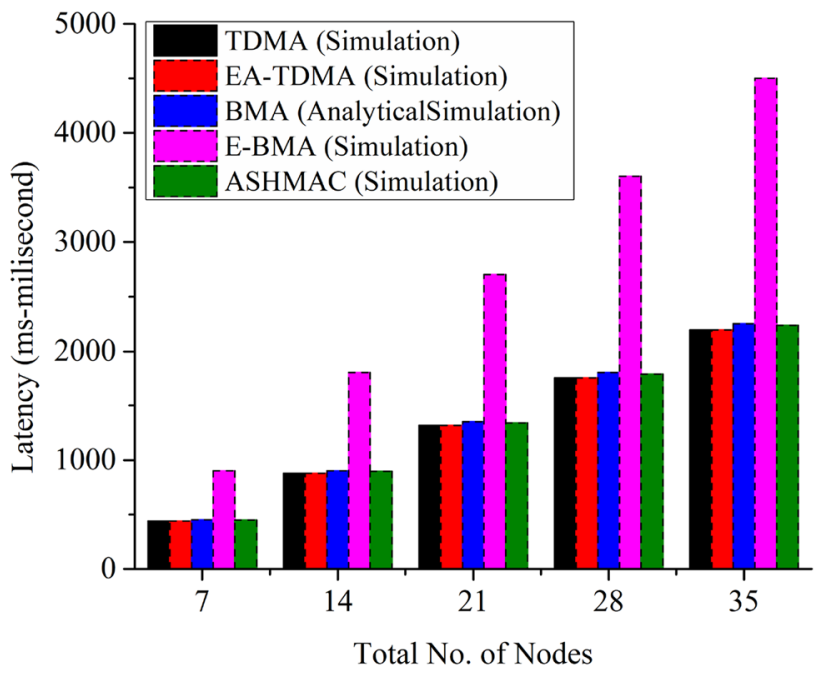

Fig. 9 Simulation results of transmission latency versus total number of nodes

\subsubsection{Transmission latency}

Maximum transmission latency is modeled, analyzed and simulated for TDMA, EA-TDMA, BMA, E-BMA and ASHMAC for different cluster sizes and different data packet sizes.

Case 1: In this case, number of nodes are varied from $N=7-35(m=2-10)$. It is assumed that data packet size is 200 bytes and $k=20$. Both analytical and simulation results show that the maximum transmission latency of E-BMA is approximately double than ASHMAC, TDMA, EATDMA and BMA as shown in Figs. 8 and 9 respectively.

Case 2: In this case, the data packet size is varied from 50 to 250 bytes. It is assumed that $N=14(m=4)$ and

\section{SN Applied Sciences}




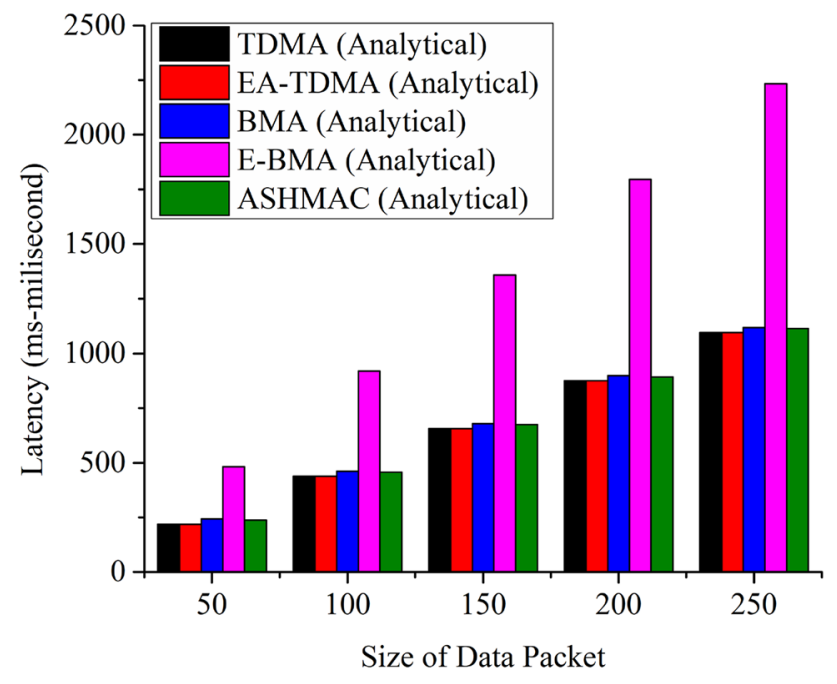

Fig. 10 Analytical results of transmission latency versus data packet size

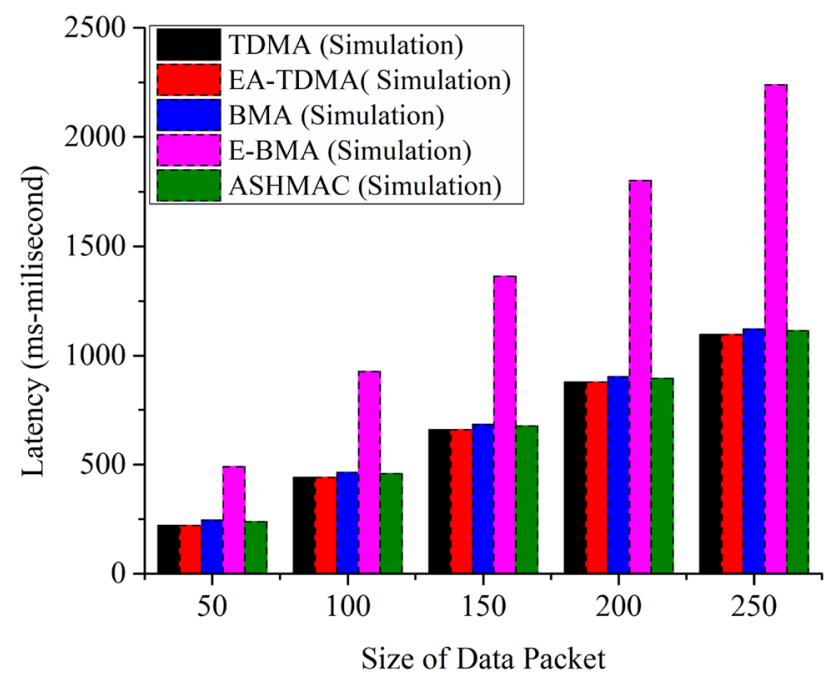

Fig. 11 Simulation results of transmission latency versus data packet size

$k=20$. Both analytical and simulation results show that maximum transmission latency of E-BMA is approximately double w.r.t. ASHMAC, TDMA, EA-TDMA and BMA as shown in Figs. 10 and 11 respectively. In the case of E-BMA, the maximum transmission latency is double due to one extra frame delay in transmission.

\subsection{Experimental analysis}

In the previously reported works, only analytical and simulation analysis has been done by the researchers for MAC layer modeling. Real-time experimental analysis is quite difficult. Because real-time scenario depends upon various other modules and protocols. The recorded data in the real-time scenario depends upon various parameters e.g. terrain profile, noise in detection of sensor data, environmental noise, etc. The real-time scenario depends upon some other analyses which are based on different other fields i.e. detection and estimation, duty cycle algorithms, clustering algorithms, intra-cluster, and inter-cluster communication, etc. These analyses are out of the scope from the present discussion on the MAC layer protocol. Therefore, we have developed a prototype for a railway condition monitoring system for analysis of our proposed MAC layer protocol. Data records of Varanasi station (State:Uttar Pradesh, Country: India) have been taken from 4th June 1:00 P.M. to 5th June 1:00 P.M. as given in Table 8. Four Arduino Uno boards are used as sensor nodes (i.e. $\mathrm{SN}-1$, SN-2, SN-3, and SN-4) and one Raspberry Pi board is used as a $\mathrm{CH}$ node. NRF24L01 module is used as a transreceiver device. All the data sensed by the sensor nodes are transmitted to the $\mathrm{CH}$ node. $\mathrm{SN}-1$ consists of an accelerometer sensor (ADXL-345) and shock-sensor (KY-002). SN-2 consists of temperature sensor DS18B20 and SN-3 consists of temperature and humidity sensor (DHT-11). SN-4 consists of a shock sensor (KY-002) and a tilt sensor (KY-020). When the train passes from the track, it activates the shock sensor, and hence $\mathrm{SN}-1$ and $\mathrm{SN}-4$ activates their accelerometer sensor and tilt sensor respectively. Accelerometer sensor $(\mathrm{SN}-1)$ collects the data for every pass of the train for 100 seconds with $100 \mathrm{~Hz}$ frequency. SN-1 transmits data in a bulk of 8 units with a packet size of 100 bytes. Each unit consists of $x, y$, and $z$ positions. Similarly, SN-2 senses the temperature at every 30 seconds, and $\mathrm{SN}-3$ senses the humidity at the same rate. $\mathrm{SN}-4$ senses the tilt on the track at every passing of the train. Accelerometer (ADXL-345) data recorded by $\mathrm{SN}-1$ is given in Fig. 12 for 10:05 P.M. to 10:40 P.M. Six trains pass from the track and their acceleration is recorded. Similarly, SN-2 and SN-3 record temperature and humidity data, respectively. The prototype model of the experimental set-up is shown in Fig. 13. Arduino boards are used for the sensor nodes and the raspberrypi board is used for $\mathrm{CH}$ node. Sensor nodes transmit the data to the $\mathrm{CH}$ node using the NRF24L01 trans-receiver. The total number of transmitted and received packets (data and control packets) by the sensor nodes and cluster head node are given in Fig. 14 for TDMA, EA-TDMA, BMA, E-BMA, and ASHMAC protocols. BMA and E-BMA transmit more number of control packets w.r.t. other protocols. In the analytical and simulation analysis, the energy consumption model is developed for the single cycle of each protocol. But, in the experimental model, WSN is analyzed for 24 hours. Therefore, for efficient analysis, the duty cycle approach is necessary for such real-time data traffic environment. A low duty cycle consumes less energy, but causes buffer-overflow and hence, 
Table 8 Records of passing of the train from the track

\begin{tabular}{cccccccccc}
\hline Time & Train no. & Time & Train no. & Time & Train no. & Time & Train no. & Time & Train no. \\
\hline $13: 35$ & 15159 & $18: 50$ & 54291 & $23: 40$ & 12332 & $5: 45$ & 12382 & $9: 30$ & 13005 \\
$13: 54$ & 14214 & $19: 00$ & 13484 & $23: 45$ & 14228 & $6: 00$ & 16359 & $9: 35$ & 18611 \\
$14: 00$ & 14257 & $19: 30$ & 12371 & $0: 40$ & 13308 & $6: 10$ & 54333 & $9: 37$ & 54292 \\
$14: 08$ & 14204 & $19: 40$ & 15022 & $1: 10$ & 15003 & $6: 13$ & 15104 & $9: 40$ & 12333 \\
$14: 35$ & 75111 & $20: 00$ & 4998 & $1: 30$ & 63553 & $6: 15$ & 4206 & $10: 15$ & 18103 \\
$14: 50$ & 14213 & $20: 10$ & 13050 & $1: 45$ & 12561 & $6: 40$ & 13258 & $10: 25$ & 12168 \\
$15: 05$ & 14015 & $20: 15$ & 13257 & $2: 00$ & 12670 & $6: 45$ & 15054 & $10: 30$ & 14236 \\
$15: 20$ & 12791 & $20: 30$ & 14224 & $2: 05$ & 13152 & $6: 55$ & 15125 & $10: 41$ & 75106 \\
$15: 25$ & 12391 & $20: 45$ & 13010 & $2: 10$ & 11062 & $7: 05$ & 13307 & $11: 20$ & 11094 \\
$15: 35$ & 18312 & $20: 55$ & 15232 & $2: 20$ & 12358 & $7: 10$ & 14006 & $11: 35$ & 1102 \\
$15: 40$ & 75112 & $21: 00$ & 11071 & $2: 25$ & 15053 & $7: 20$ & 54263 & $11: 45$ & 19045 \\
$15: 50$ & 5092 & $21: 16$ & 13316 & $2: 50$ & 54334 & $7: 25$ & 12165 & $12: 00$ & 15018 \\
$15: 55$ & 11072 & $21: 38$ & 15231 & $3: 00$ & 54266 & $7: 50$ & 75115 & $12: 10$ & 13009 \\
$16: 05$ & 14253 & $21: 40$ & 13238 & $3: 30$ & 19421 & $7: 55$ & 55122 & $12: 30$ & 12355 \\
$16: 10$ & 11061 & $22: 00$ & 11046 & $3: 50$ & 23392 & $8: 10$ & 3427 & $12: 35$ & 15159 \\
$16: 20$ & 14203 & $22: 05$ & 4997 & $4: 00$ & 12369 & $8: 25$ & 9019 & $12: 40$ & 54264 \\
$16: 40$ & 19322 & $22: 13$ & 15126 & $4: 05$ & 12167 & $8: 30$ & 14265 & $12: 55$ & 13413 \\
$16: 55$ & 13239 & $22: 17$ & 54256 & $4: 15$ & 63554 & $8: 35$ & 22418 & $13: 15$ & 11107 \\
$17: 00$ & 12792 & $22: 23$ & 14220 & $4: 30$ & 19052 & $8: 40$ & 13134 & $13: 40$ & 12237 \\
$17: 10$ & 54265 & $22: 28$ & 75116 & $4: 45$ & 24227 & $8: 50$ & 13049 & $14: 00$ & 12537 \\
$17: 25$ & 14853 & $22: 35$ & 13119 & $4: 50$ & 14258 & $8: 55$ & 14223 & $14: 28$ & 14204 \\
$17: 45$ & 11108 & $23: 00$ & 53525 & $4: 55$ & 11093 & $9: 00$ & 1101 & $14: 45$ & 13346 \\
$17: 50$ & 14005 & $23: 08$ & 15103 & $5: 00$ & 13151 & $9: 02$ & 22408 & $14: 45$ & 13346 \\
\hline & & & & & & & & &
\end{tabular}

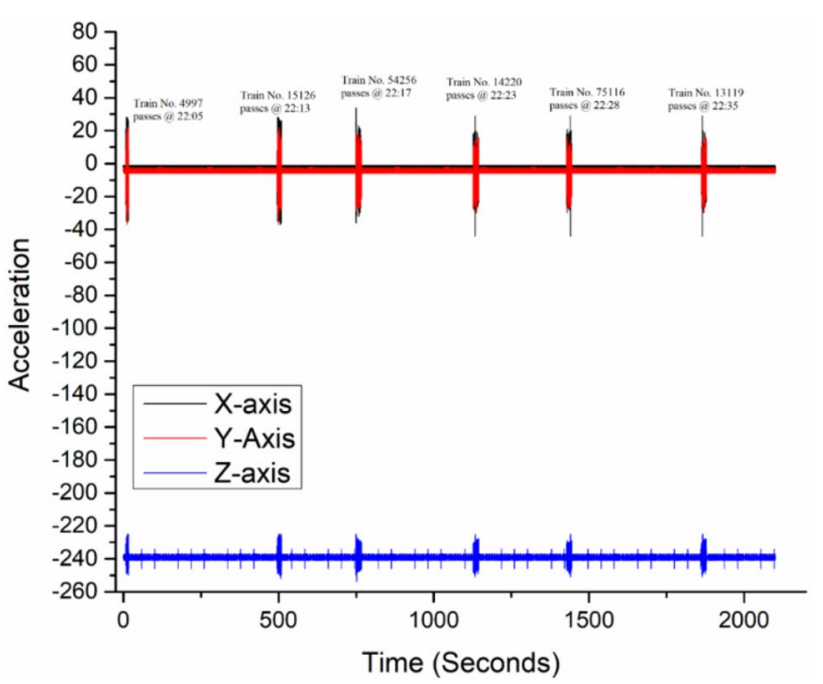

Fig. 12 Accelerometer recorded data from ADXL-345 for X-axis, $\mathrm{Y}$-axis and Z-axis

throughput reduces. Therefore, in the present analysis, the optimum duty cycle is considered for the analysis purpose. As shown in Fig. 15 that below $17 \%$ of duty cycle performance degrades, buffer-overflow increases, and hence throughput decreases. Therefore, in the present work, a $17 \%$ duty cycle is taken as optimum value.

Figure 16 shows that the energy consumption of ASHMAC is quite less than TDMA, EA-TDMA, and BMA MAC protocols and approximately equal to the E-BMA MAC protocol. Figure 17 shows that the maximum transmission latency of ASHMAC is quite lesser than E-BMA. Therefore, the optimal performance of our proposed protocol is much better than existing MAC protocols.

From the above analysis, overall summary of the results is as follows:

- In all the cases, ASHMAC is more energy-efficient than TDMA, EA-TDMA, and BMA for low, medium, and high traffic but slightly less energy efficient than E-BMA.

- In terms of transmission latency, ASHMAC is far better than E-BMA.

- Overall optimized performance of ASHMAC is much better than BMA, TDMA, EA-TDMA, and E-BMA.

- For the validation of the simulation and analytical results, an experimental prototype model has also been developed. The experimental results prove the validity of our proposed model. 
Fig. 13 Block diagram of experimental set-up

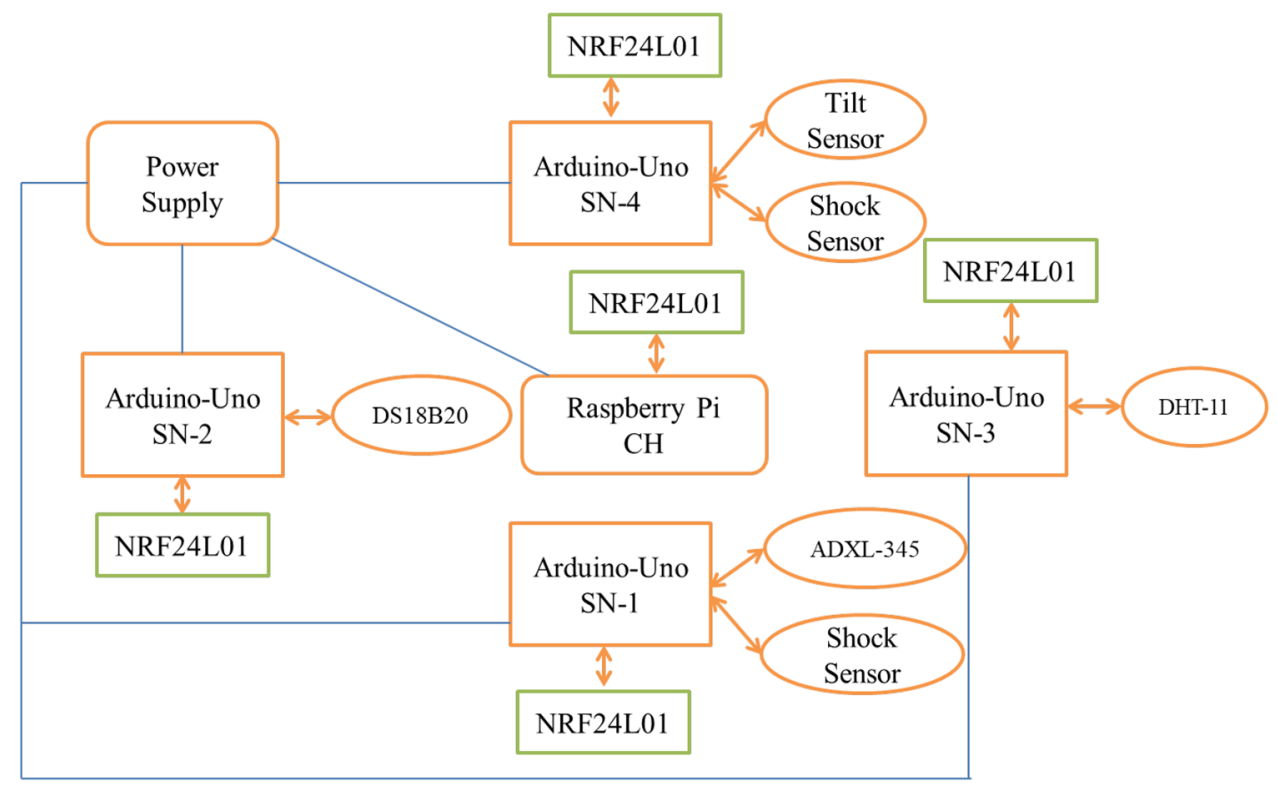

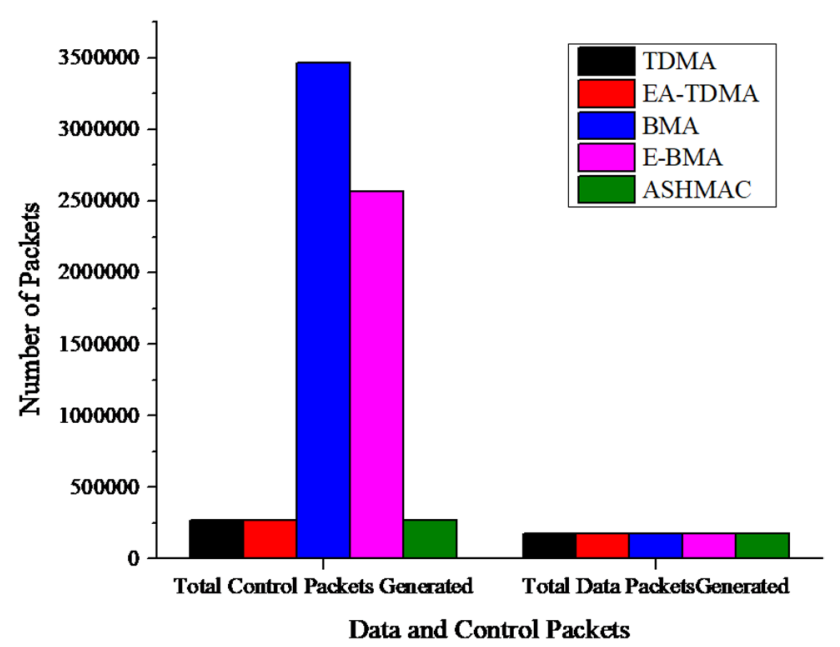

Fig. 14 Number of control and data packets transmitted by various protocols

\section{Conclusion}

An improved application-specific hybrid medium access control (ASHMAC) protocol is proposed for railway monitoring applications. The ASHMAC saves unnecessary energy loss, which occurs in the BMA MAC protocol for continuous monitoring nodes due to the contention period. It also saves unnecessary wastage of energy, which occurs in TDMA MAC protocol for event-driven nodes due to reservation of data slots to the non-source nodes. Comparative performance analysis is done with other existing MAC protocols. ASHMAC is a hybrid MAC protocol that includes the features of both TDMA and

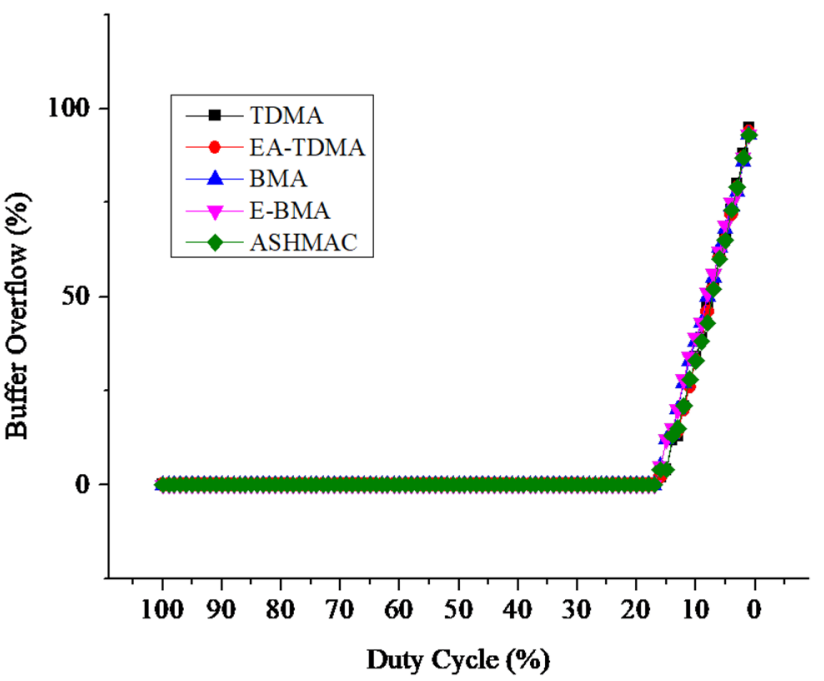

Fig. 15 Buffer-overflow versus duty cycle

BMA. Simulation and analytical models have been developed and proposed for ASHMAC protocol and its performance is compared with TDMA, EA-TDMA, BMA, and E-BMA protocols. The results show the effectiveness of the proposed protocol for both event-driven and continuous monitoring applications. Analytical and simulation results show that ASHMAC performs better than TDMA, EA-TDMA, and BMA for low and medium traffic in terms of energy efficiency. The drawback of ASHMAC is that it is slightly less energy efficient than E-BMA. However, the transmission latency of E-BMA is around two times higher than ASHMAC. Therefore, the overall optimal performance of ASHMAC is better than the other mentioned protocols for low, medium, and high data traffic 


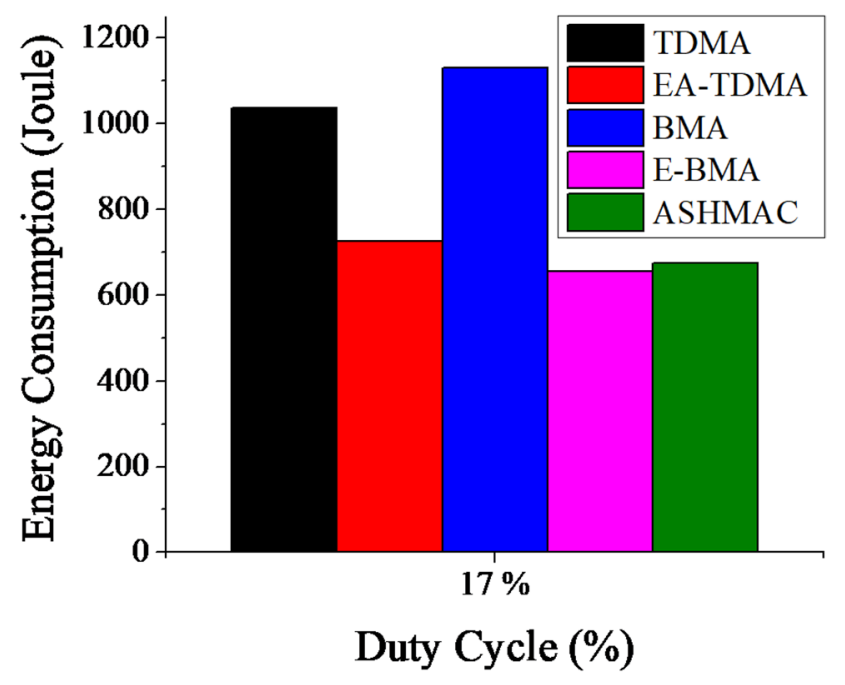

Fig. 16 Experimental results of energy consumption

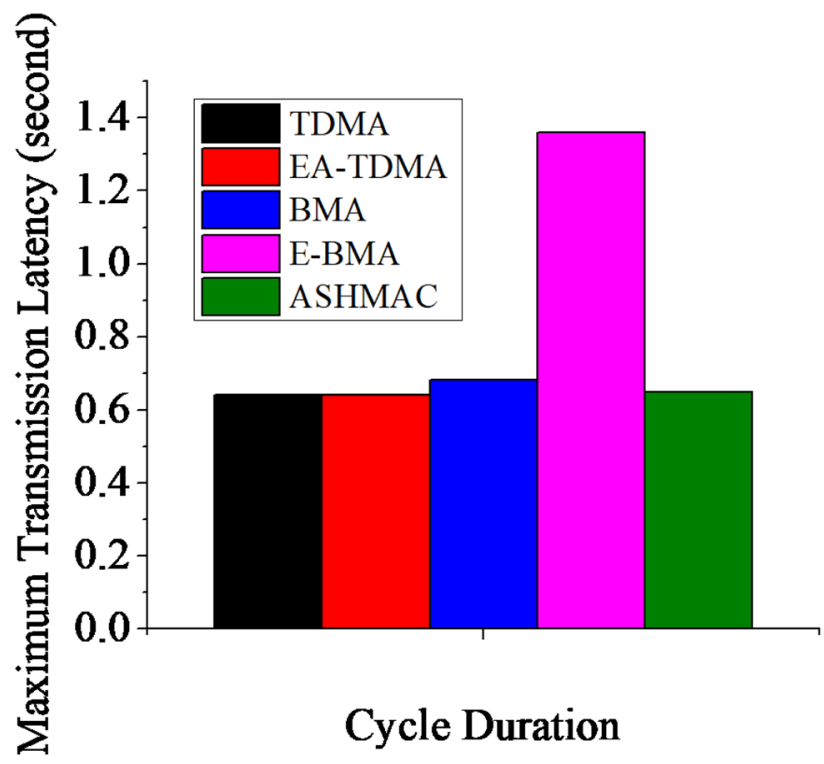

Fig. 17 Experimental results of maximum transmission latency

applications. In the future, we plan to extend our work by hybridization of EA-TDMA/E-BMA instead of TDMA/ BMA. E-BMA consumes less energy than BMA even at high data traffic load conditions. However, E-BMA follows piggybacking for reservation of consecutive data slots which is significantly challenging to implement in hybrid MAC protocol.

\section{Compliance with ethical standards}

Conflict of interest The authors declare that they have no conflict of interest.

\section{References}

1. Hodge VJ, O'Keefe S, Weeks M, Moulds A (2015) Wireless sensor networks for condition monitoring in the railway industry: a survey. IEEE Trans Intell Transp Syst 16(3):1088-1106

2. Aw E (2007) Low cost monitoring system to diagnose problematic rail bed: case study of mud pumping site. Ph.D. dissertation, Massachusetts Institute of Technology, Cambridge, MA, USA

3. Baum RL, Godt JW, Harp EL, McKenna JP (2005) Early warning of landslides for rail traffic between Seattle and Everett. In: Proceedings of the landslide risk management, Washington, USA, pp 731-740

4. Anastasopoulos A, Bollas K, Papasalouros D, Kourousis D (2010) Acoustic emission on-line inspection of rail wheels. In: Proceedings of the 29th European conference on acoustic emission testing, Wien, Austria, pp 1-8

5. Thakkar N, Steel JA, Reuben RL (2010) Rail-wheel interaction monitoring using acoustic emission: a laboratory study of normal rolling signals with natural rail defects. Mech Syst Signal Process 24(1):256-266

6. Barke D, Chiu W (2005) Structural health monitoring in the railway industry: a review. Struct Health Monit 4(1):81-93

7. Belotti V, Crenna F, Michelini R, Rossi G (2006) Wheel-flat diagnostic tool via wavelet transform. Mech Syst Signal Process 20(8):1953-1966

8. Balas V, Jain L (2010) World knowledge for sensors and estimators by models and internal models. J Intell Fuzzy Syst 21(1):79-88

9. Kolakowski P, Szelazek J, Sekula A, Swiercz K, Mizerski K, Gutkiewicz P (2011) Structural health monitoring of a railway truss bridge using vibration-based and ultrasonic methods. Smart Mater Struct 20(3):035016

10. Ye W, Heidemann J, Estrin D (2002) An energy-efficient MAC protocol for wireless sensor networks. In: Proceedings twentyfirst annual joint conference of the IEEE computer and communications societies, vol 3, pp 1567-1576

11. Stem M, Katz RH (1997) Measuring and reducing energy consumption of network interfaces in hand held devices. IEICE Trans Commun E80--B(8):1125-1131

12. Lee $A H$, Jing $M H$, Kao CY (2008) LMAC: an energy-latency trade-off MAC protocol for wireless sensor networks. In: International symposium on computer science and its applications, Hobart, ACT, pp 233-238

13. Pei G, Chien C (2001) Low power TDMA in large wireless sensor networks. In: 2001 MILCOM proceedings of the communications for network-centric operations: creating the information force (cat. no. 01CH37277), vol 1, pp 347-351

14. Shafiullah G, Azad SA, Ali ABMS (2013) Energy-efficient wireless MAC protocols for railway monitoring applications. IEEE Trans Intell Transp Syst 14(2):649-659

15. Shafiullah GM, Thompson A, Wolf P, Ali S (2008) Energy-efficient TDMA MAC protocol for wireless sensor networks applications. In: Proceedings of the 5th ICECE, Dhaka, Bangladesh, Dec 24-27, pp 85-90

16. Li J, Lazarou GY (2004) A bit-map-assisted energy-efficient MAC scheme for wireless sensor networks. In: Third international symposium on information processing in sensor networks. IPSN 2004, pp 55-60

17. Heinzelman WB, Chandrakasan AP, Balakrishnan H (2002) An application-specific protocol architecture for wireless microsensor networks. IEEE Wirel Commun Trans 1(4):660-670

18. Wang L et al (2018) A hybrid TDMA/CSMA-based wireless sensor and data transmission network for ORS intra-microsatellite applications. Sensors (Basel, Switzerland) 18(5):1537 
19. Du S, Sun Y, Johnson DB (2010) EMAC: an asynchronous routing-enhanced MAC protocol in multi-hop wireless networks. In: IEEE global telecommunications conference GLOBECOM 2010, Miami, FL, pp 1-6

20. Hoesel LFW, Havinga PJM (2004) A lightweight medium access protocol (LMAC) for wireless sensor networks: reducing preamble transmissions and transceiver state switches. In: 1st international workshop on networked sensing systems, pp 205-208

21. Chatterjea S, van Hoesel LFW, Havinga PJM (2004) AI-LMAC: an adaptive, information-centric and lightweight MAC protocol for wireless sensor networks. In: First international conference on intelligent sensors, sensor networks and information processing, pp 381-388

22. Rhee I, Warrier A, Aia M, Min J, Sichitiu ML (2008) Z-MAC: a hybrid MAC for wireless sensor networks. IEEE/ACM Trans Netw 16(3):511-524

23. Alvi AN, Bouk SH, Ahmed SH, Yaqub MA, Sarkar M, Song H (2016) BEST-MAC: bitmap-assisted efficient and scalable TDMA-based WSN MAC protocol for smart cities. IEEE Access 4:312-322

24. An IEEE 802.15.4 complaint and ZigBee-ready $2.4 \mathrm{GHz}$ RF transceiver. Microw J 47(6):130-135 (2004)

25. Philipose A, Rajesh A (2015) Performance analysis of an improved energy aware MAC protocol for railway systems. In: 2015 2nd international conference on electronics and communication systems (ICECS), Coimbatore, pp 233-236

26. Kumar D, Singh MP (2018) Bit-map-assisted energy-efficient MAC protocol for wireless sensor networks. Int J Adv Sci Technol 119:111-122
27. Tolani M, Sunny, Singh RK, Shubham K, Kumar R (2019) Energy efficient adaptive bit-map-assisted medium access control protocol. In: Wireless personal communication. Springer

28. Tolani M, Sunny, Singh RK (2020) Adaptive duty-cycle-enabled energy-efficient bit-map-assisted MAC protocol. SN Comput Sci :144. https://doi.org/10.1007/s42979-020-00162-7

29. Dargie W, Poellabauer C (2010) Fundamentals of wireless sensor networks: theory and practice. Wiley, New York

30. Kumar S, Kim H (2020) BH-MAC: an efficient hybrid MAC protocol for vehicular communication. In: 2020 international conference on communication systems \& networks (COMSNETS), Bengaluru, India, pp 362-367. https://doi.org/10.1109/COMSNETS48 256.2020.9027322.

31. Tolani M, Sunny, Singh RK (2019) Lifetime improvement of wireless sensor network by information sensitive aggregation method for railway condition monitoring. Ad Hoc Netw 8:128-145

Publisher's Note Springer Nature remains neutral with regard to jurisdictional claims in published maps and institutional affiliations. 